\title{
The AD775 cosmic event revisited: the Sun is to blame
}

\author{
I. G. Usoskin ${ }^{1}$, B. Kromer ${ }^{2}$, F. Ludlow ${ }^{3}$, J. Beer ${ }^{4}$, M. Friedrich ${ }^{5}$, G. A. Kovaltsov ${ }^{6}$, S. K. Solanki ${ }^{7,8}$, and L. Wacker ${ }^{9}$ \\ 1 Sodankylä Geophysical Observatory (Oulu unit) and Physics Dept., University of Oulu, 99600 Sodankylä, Finland \\ e-mail: Ilya.Usoskin@oulu.fi \\ 2 Klaus-Tschira-Laboratory for Scientific Dating, Curt-Engelhorn-Centre for Archaeometry, D6, 3, 68159 Mannheim, Germany \\ 3 Harvard University Center for the Environment, and Department of History, Harvard University, Cambridge, MA 02138, USA \\ 4 Swiss Federal Institute of Aquatic Science and Technology, Eawag, Überlandstrasse 133, 8600 Dübendorf, Switzerland \\ 5 Hohenheim University, Institute of Botany (210), 70593 Stuttgart, Germany \\ ${ }^{6}$ Ioffe Physical-Technical Institute, 194021 St. Petersburg, Russia \\ 7 Max Planck Institute for Solar System Research, Max-Planck-Str. 2, 37191 Katlenburg-Lindau, Germany \\ 8 School of Space Research, Kyung Hee University, Yongin, 446-701 Gyeonggi, Korea \\ 9 Department of Physics, Swiss Federal Institute of Technology ETHZ, 8092 Zurich, Switzerland
}

Received 11 January 2013 / Accepted 26 February 2013

\begin{abstract}
Aims. Miyake et al. (2012, Nature, 486, 240, henceforth M12) recently reported, based on ${ }^{14} \mathrm{C}$ data, an extreme cosmic event in about AD775. Using a simple model, M12 claimed that the event was too strong to be caused by a solar flare within the standard theory. This implied a new paradigm of either an impossibly strong solar flare or a very strong cosmic ray event of unknown origin that occurred around AD775. However, as we show, the strength of the event was significantly overestimated by M12. Several subsequent works have attempted to find a possible exotic source for such an event, including a giant cometary impact upon the Sun or a gamma-ray burst, but they are all based on incorrect estimates by M12. We revisit this event with analysis of new datasets and consistent theoretical modelling.

Methods. We verified the experimental result for the AD775 cosmic ray event using independent datasets including ${ }^{10} \mathrm{Be}$ series and newly measured ${ }^{14} \mathrm{C}$ annual data. We surveyed available historical chronicles for astronomical observations for the period around the AD770s to identify potential sightings of aurorae borealis and supernovae. We interpreted the ${ }^{14} \mathrm{C}$ measurements using an appropriate carbon cycle model.

Results. We show that: (1) The reality of the AD775 event is confirmed by new measurements of ${ }^{14} \mathrm{C}$ in German oak; (2) by using an inappropriate carbon cycle model, M12 strongly overestimated the event's strength; (3) the revised magnitude of the event (the global ${ }^{14} \mathrm{C}$ production $Q=(1.1-1.5) \times 10^{8}$ atoms $\left./ \mathrm{cm}^{2}\right)$ is consistent with different independent datasets $\left({ }^{14} \mathrm{C},{ }^{10} \mathrm{Be}\right.$, $\left.{ }^{36} \mathrm{Cl}\right)$ and can be associated with a strong, but not inexplicably strong, solar energetic particle event (or a sequence of events), and provides the first definite evidence for an event of this magnitude (the fluence $>30 \mathrm{MeV}$ was about $4.5 \times 10^{10} \mathrm{~cm}^{-2}$ ) in multiple datasets; (4) this interpretation is in agreement with increased auroral activity identified in historical chronicles.

Conclusions. The results point to the likely solar origin of the event, which is now identified as the greatest solar event on a multimillennial time scale, placing a strong observational constraint on the theory of explosive energy releases on the Sun and cool stars.
\end{abstract}

Key words. Sun: activity - Sun: flares

\section{Introduction}

It is of particular interest, both from astrophysical and societal points of view, to understand the full spectrum of severity of extreme events in the Earth's radiation environment. In particular, knowing the maximum possible energy of solar energetic particle (SEP) events and the frequency of their occurrence is of great importance for solar and stellar physics (Hudson 2010). The history of direct solar observations is relatively short, spanning only several decades and provides insufficient statistics on the occurrence rate of the most energetic SEP events (Smart et al. 2006). Instead, long-term records of cosmogenic isotopes, like ${ }^{14} \mathrm{C}$ and ${ }^{10} \mathrm{Be}$ stored in terrestrial, meteoritic, or lunar archives, can reveal the history of such events over a greatly extended time-span (Usoskin 2008; Beer et al. 2012). While the statistics of extreme SEP events remains unclear to some extent (Hudson 2010), progress in this field have been recently made (Schrijver et al. 2012; Usoskin \& Kovaltsov 2012) allowing the occurrence frequency of such events to be better estimated by joint analysis of different cosmogenic isotope data.

A particularly exciting result has been recently published by Miyake et al. (2012, henceforth M12) who found a significant enhancement of about $1.5 \%$ (15 permill) of ${ }^{14} \mathrm{C}$ content measured in Japanese cedars around AD775. Using a basic four-box model of the carbon cycle, M12 estimated the corresponding absolute global ${ }^{14} \mathrm{C}$ production for the event as $6 \times$ $10^{8}$ atoms $/ \mathrm{cm}^{2}$ or 19 atoms $/ \mathrm{cm}^{2} / \mathrm{s}$ averaged over a year. This is an order of magnitude greater than the average ${ }^{14} \mathrm{C}$ production rate due to galactic cosmic rays (GCR), which is estimated to be $1.6-2$ atoms $/ \mathrm{cm}^{2} / \mathrm{s}$ (Kovaltsov et al. 2012, and references therein) for the pre-industrial era. When translating the production rate into the flux of cosmic rays or the energy of the source, candidates being either a giant solar eruption or a nearby supernova, M12 concluded that it was much too high, implying a sudden strong cosmic ray event of unknown origin. In an attempt to resolve the situation Allen (2012) proposed "a supernova largely 


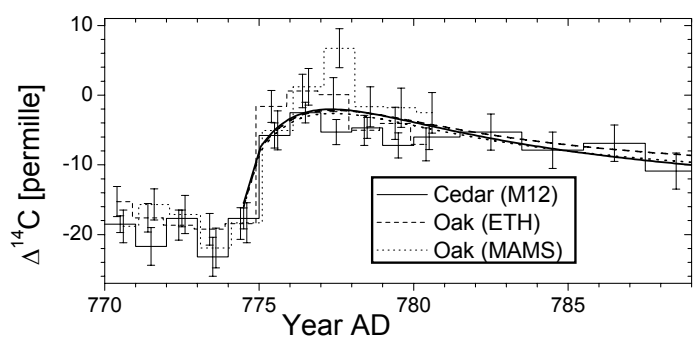

Fig. 1. Time profiles of the measured $\Delta^{14} \mathrm{C}$ content in Japanese cedar (Miyake et al. 2012) and German oak (this work) for the period around AD775. Smooth black and grey lines depict a family of best fit $\Delta^{14} \mathrm{C}$ profiles, calculated using a family of realistic carbon cycle models for an instantaneous injection of ${ }^{14} \mathrm{C}$ into the stratosphere.

hidden behind a dust cloud... The resulting supernova remnant would be invisible". However as discussed below, this interpretation is unlikely. Recently Melott \& Thomas (2012) recalculated the energy of a possible coronal mass ejection (CME) related to such a burst of SEPs and found a lower value. But it is still too energetic to correspond to a realistic solar eruptive event, leaving open the problem of the possible source of the AD775 event. Eichler \& Mordecai (2012) tried to link the event source to an impact of a cometary body upon the Sun producing a shock in the corona to sufficiently accelerate energetic particles. Hambaryan \& Neuhäuser (2013) proposed a gamma-ray burst as a possible source. Thus, a number of exotic sources have been proposed, because the most plausible one, the SEP event, looks unrealistically energetic given the strength of the event as estimated by M12. Instead, the M12 result disagrees with data on another cosmogenic radionuclide, ${ }^{10} \mathrm{Be}$ in the Dome Fuji ice core (Horiuchi et al. 2008). The two cosmogenic isotopes $\left({ }^{14} \mathrm{C}\right.$ and ${ }^{10} \mathrm{Be}$ ) are formed as sub-products of the same process of a nucleonic-electromagnetic-muon cascade caused by energetic cosmic rays or $\gamma$-quanta in the Earth's atmosphere (e.g., Beer et al. 2012). Thus, it is hardly possible to obtain a tenfold increase in the annual ${ }^{14} \mathrm{C}$ production without a corresponding increase in ${ }^{10} \mathrm{Be}$ data (Usoskin et al. 2006). This indicates an inconsistency in the scenario proposed by M12. This event was also analyzed by Usoskin \& Kovaltsov (2012) who found the strength of the event much lower than did M12. Here we revisit the AD775 event with analysis of new datasets and consistent theoretical modelling.

\section{2. ${ }^{14} \mathrm{C}$ in German oak}

In order to verify the existence and strength of the claimed AD775 event, annual samples from a German oak (tree Steinbach 91 from the river Main), a part of the German Oak Chronology (Friedrich et al. 2004), were measured independently in facilities of Mannheim, Germany (MAMS) and the ETH Zurich, Switzerland (ETH) for the period AD770-780. The data (Fig. 1) are presented here for the first time. One can see that they confirm the increase of ${ }^{14} \mathrm{C}$ production around $\mathrm{AD} 775$ found by M12. Despite a possible slight vertical offset explainable by local peculiarities, the magnitude and timing of the increase are in full agreement. This implies that the event was global and was caused by the enhanced production of ${ }^{14} \mathrm{C}$.

\section{Analysis of ${ }^{14} \mathrm{C}$ data}

Only the relative content of ${ }^{14} \mathrm{C}$ is measured in the atmosphere. In order to evaluate the ${ }^{14} \mathrm{C}$ production, and hence the strength of

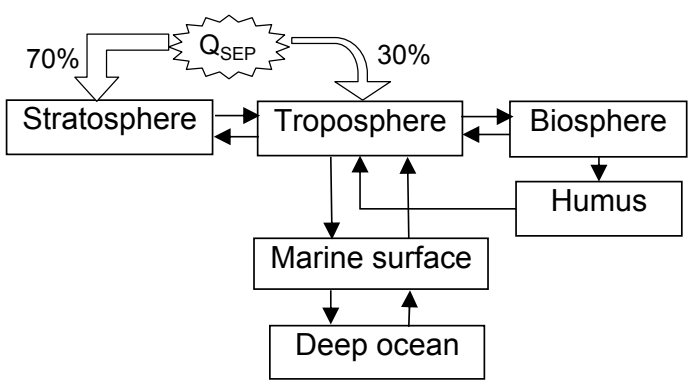

Fig. 2. A scheme of the carbon cycle models used here with arrows depicting the carbon exchange between different reservoirs. Big arrows denote production $Q$ of ${ }^{14} \mathrm{C}$ in the atmosphere.

the event, one needs to run a carbon cycle model and to compare the relative increase of $\Delta^{14} \mathrm{C}$ with the background concentration of ${ }^{14} \mathrm{C}$ in the troposphere, from where it is absorbed by living trees. Here we use a family of three carbon cycle models, including the main carbon reservoirs (Fig. 2): (1) a five-box model (Dorman 2004, page 703); (2) a five-box model (Dergachev \& Veksler 1991; Damon \& Peristykh 2004); and (3) a box-diffusion model (Oeschger et al. 1975; Siegenthaler et al. 1980). For better modeling of the SEP signal we also divided the atmosphere into stratosphere and troposphere, with a stratospheric carbon capacity of 15\% (cf. Miyake et al. 2012) and a residence time of two years (Damon et al. 1978). A model (Kovaltsov et al. 2012) was used to simulate the ${ }^{14} \mathrm{C}$ production by SEPs with a hard energy spectrum, assuming $70 \%$ of ${ }^{14} \mathrm{C}$ produced by SEPs in the stratosphere and $30 \%$ in the troposphere, globally. These carbon cycle models differ slightly in exchange times between reservoirs and in dealing with the ocean, but they yield very similar results for the expected ${ }^{14} \mathrm{C}$ signal (Fig. 1). The background ${ }^{14} \mathrm{C}$ level was taken as the mean pre-industrial production rate of 1.6 atoms $/ \mathrm{cm}^{2} / \mathrm{s}$ (Goslar 2001). The best fit by the weighted least-square method between the three datasets and the three models yields a net ${ }^{14} \mathrm{C}$ production of $(1.3 \pm 0.2) \times$ $10^{8}$ atoms $/ \mathrm{cm}^{2}$. This is a factor of about 5 smaller than the M12 model $\left(6 \times 10^{8}\right.$ atoms $\left./ \mathrm{cm}^{2}\right)$. M12 used a different carbon cycle model, neglecting the deep ocean (Fig. 2), which is the greatest reservoir containing $92-95 \%$ of all carbon. Thus, M12 greatly overestimated the background ${ }^{14} \mathrm{C}$ concentration in the troposphere. This led to an error when translating the relative increase of $\Delta{ }^{14} \mathrm{C}$ into the ${ }^{14} \mathrm{C}$ production and so the event strength. We conclude that M12 overestimated the production by a factor of 4-6 because of the inappropriate model. Accordingly, all energy/particle flux estimates based on the numbers given by M12 are incorrect by the same factor.

We propose that the total ${ }^{14} \mathrm{C}$ production for the $\mathrm{AD} 775$ event corresponds to a SEP fluence $(>30 \mathrm{MeV})$ of $F_{30} \approx$ $4.5 \times 10^{10} \mathrm{~cm}^{-2}$, with a hard spectrum as per the SEP event of 23 Feb. 1956 (cf. Usoskin \& Kovaltsov 2012). Since ${ }^{14} \mathrm{C}$ is produced by more energetic particles, the value of $F_{30}$ depends on the assumed spectrum. For example, by assuming a very soft SEP spectrum as per the event of Aug. 1972, one would obtain the $F_{30}$ fluence $1.8 \times 10^{11} \mathrm{~cm}^{-2}$. More robust is the fluence of SEP with energy $>200 \mathrm{MeV}$, which is $(5.5-11) \times 10^{9} \mathrm{~cm}^{-2}$ for our scenario irrespective of the assumed SEP energy spectrum.

In addition to the annual ${ }^{14} \mathrm{C}$ measurements in individual trees, discussed above, we use a five-year averaged INTCAL09 global ${ }^{14} \mathrm{C}$ series (Reimer et al. 2009) (Fig. 3A). The proposed scenario is consistent with the data $\left(\chi^{2} /\right.$ d.o.f. $\left.=0.68\right)$. A time shift of a few years can be explained by the filtering of the raw data in the INTCAL09 series (Hogg et al. 2009). 


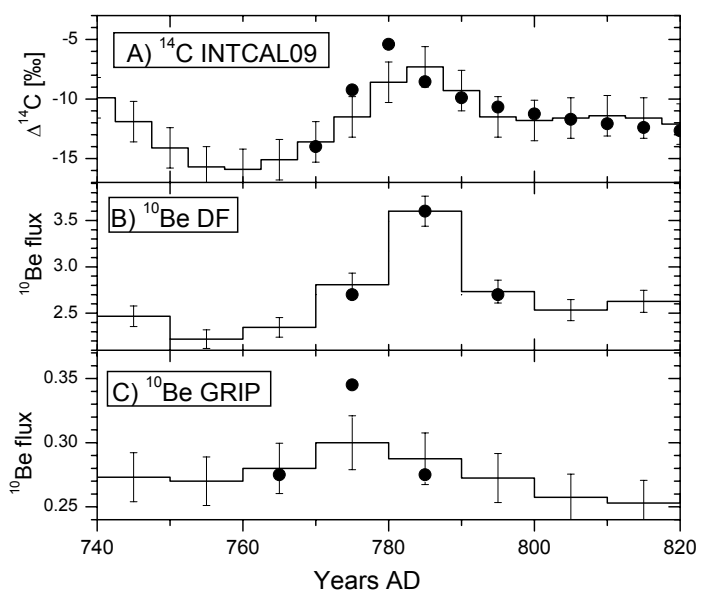

Fig. 3. Time profiles of the measured cosmogenic isotopes (histograms with error bars) for the period around AD775. Solid dots represent the enhancement expected in each data series assuming the AD775 event scenario discussed here $\left({ }^{14} \mathrm{C}\right.$ production $\left.Q=1.3 \times 10^{8} \mathrm{at} / \mathrm{cm}^{2}\right)$. A) INTCAL09 5-yr samples global atmospheric $\Delta^{14} \mathrm{C}$ (Reimer et al. 2009); B) quasi-decadal ${ }^{10} \mathrm{Be}$ content in the Dome Fuji ice core (Horiuchi et al. 2008), our model data is shifted by 5 years to match the observed data; C) quasi-decadal ${ }^{10} \mathrm{Be}$ content in the GRIP ice core (Yiou et al. 1997).

\section{4. ${ }^{10} \mathrm{Be}$ in ice cores}

Using the above scenario, we calculated the expected increase in ${ }^{10} \mathrm{Be}$ by applying the production model of Kovaltsov \& Usoskin (2010) and assuming an instant injection and intermediate atmospheric mixing of ${ }^{10} \mathrm{Be}$ (McCracken 2004; Vonmoos et al. 2006). Note that the ratio of the production of ${ }^{14} \mathrm{C}$ and ${ }^{10} \mathrm{Be}$ by a SEP event is almost independent of the assumption of the SEP spectrum (Usoskin et al. 2006). Here we analyze two ${ }^{10}$ Be series measured in ice cores. One is the Antarctic Dome Fuji series (Horiuchi et al. 2007, 2008) (Fig. 3B). The proposed scenario perfectly fits the data by magnitude, but the peak is delayed by several years and formally appears after AD780. However, since the dating of the ${ }^{10} \mathrm{Be}$ series is related to the resolution of the stratigraphic intervals sampled and is dependent on an age-depth model between tie points, dating uncertainties of several years are possible in the ice-core datasets (Beer 2000; Horiuchi et al. 2007). Thus, our proposed scenario is totally consistent with the ${ }^{10} \mathrm{Be}$ data measured in the Antarctic Dome Fuji ice core. The other dataset is the Greenland GRIP series (Yiou et al. 1997; Vonmoos et al. 2006) (Fig. 3C). There is a weak increase during the AD770s but it is not pronounced. The proposed scenario yields an expected peak that is higher (by about $2 \sigma$ ) than the observed peak. Thus, the GRIP series is not fully consistent with our scenario and the other data series, but the existence of the peak cannot be excluded at the $5 \%$ level. Instead, the corresponding ${ }^{36} \mathrm{Cl}$ peak in the GRIP core ${ }^{1}$ agrees with the proposed scenario.

\section{Historical observations of the Aurora Borealis}

We surveyed published aurora catalogues from oriental chronicles at low latitudes. Keimatsu (1973) and Yau et al. (1995) cite credible observations from Shanxi Province, China, in AD770 (twice), AD773, and AD775. The next nearest observations are at AD767 and AD786. We also survey catalogues from occidental chronicles. Link (1962) cites the "red cross"

\footnotetext{
1 Estimate by JB based on unpublished preliminary data.
}

in the sky dated AD773/774 in different manuscripts of the Anglo-Saxon Chronicle (England), and "inflamed shields" in the sky (Germany, AD776). The next nearest occidental observations are at AD765 and AD786. At this time the Bible was a key reference in interpreting natural phenomena, explaining the cryptic reporting of aurorae (e.g., the above cited "red cross", also translated as "red sign of Christ" by Swanton 2000). In a new survey of occidental chronicles, we identified probable aurorae in AD772 ("fire from heaven", Ireland) and in an AD773 apparition interpreted by Christians as riders on white horses (Germany). Further new Irish observations are dated AD765 and AD786, and are found in the Annals of Ulster (Mac Airt \& Mac Niocaill 1983), and the above-cited AD773 report from Germany in the Royal Frankish Annals (Scholz \& Rogers 1970). We surveyed historical nova catalogues (e.g., Xu et al. 2000) but found no credible report of supernovae in the AD770s. Allen (2012) interprets the red cross (Anglo-Saxon Chronicle) as an exotic nearby supernova with an unobservable remnant, but we interpret this as an aurora. This is supported by a report that the same year "snakes... seen extraordinarily in the land of the South-Saxons" (Swanton 2000). Serpents often feature in descriptions of aurorae (Dall'Olmo 1980), reflecting the sinuous movement of auroral structures. We also note the uncertain dating of these events in the Anglo Saxon Chronicle, with Swanton (2000) re-dating them to AD776, i.e., after the onset of the AD775 event. We do not directly associate any particular aurora with the ${ }^{14} \mathrm{C}$ event, but a distinct cluster of aurorae between AD770 and AD776 suggests a high solar activity level around AD775. With the next nearest observations around AD765-767 and AD786, this suggests an 11-year cyclicity.

\section{Discussion and conclusions}

The existence of the AD775 event is confirmed using a larger dataset, including two new annual ${ }^{14} \mathrm{C}$ series from German oak (Fig. 1), but the event's interpretation by M12 is found to be incorrect. Because of an inappropriate carbon cycle model, M12 strongly overestimated (by a factor of 4-6) the strength of the event. Here we re-conducted the analysis, using more realistic models. The consequent event-integrated ${ }^{14} \mathrm{C}$ production rate is $(1.1-1.5) \times 10^{8}$ atoms $/ \mathrm{cm}^{2}$. We have verified that this value is in agreement with all the considered data series, including ${ }^{10} \mathrm{Be}$ records in polar ice, yielding a consistent view corresponding to a strong SEP event (or a sequence of events) with a hard energy spectrum, 25-50 times stronger than the SPE event of 23 Feb. 1956 (cf. Usoskin \& Kovaltsov 2012). Such an event, while very strong, is not impossible for the solar dynamo (Hudson 2010). Moreover, it can be a sequence of SEP events as, for example, happened in the Autumn of 1989, thus further reducing the severity of individual events. This is corroborated by the steep tail of the SEP event fluence distribution (Schrijver et al. 2012). Several potential candidates for similar events have previously been identified (Usoskin \& Kovaltsov 2012), but observed in single data series only, thus leaving room for a possible terrestrial origin (e.g. regional climate excursion). The AD775 event is the only one consistently observed in several independent datasets, thus providing the first unequivocal observational evidence of such a strong SEP event on a multi-millennial time scale. This places a strong observational constraint on the upper limits of solar eruptive events, which is important for solar and more broadly stellar physics.

In conclusion, by correcting the M12 model, by providing new independent ${ }^{14} \mathrm{C}$ data, and by surveying available historical chronicles and published aurora catalogues, we revisited the 
AD775 event to demonstrate that it can likely be attributed to a strong solar SEP event. We show that:

- The existence of the AD775 event is confirmed by new measurements of ${ }^{14} \mathrm{C}$ in German oak and by the existing ${ }^{10} \mathrm{Be}$ data from polar ice cores.

- Miyake et al. (2012) overestimated the event's strength by a factor of 4-6. This directly affects subsequent works based on this incorrect estimate (e.g., Melott \& Thomas 2012; Eichler \& Mordecai 2012; Hambaryan \& Neuhäuser 2013).

- The revised event is consistent with different independent datasets and is associated with a strong, but not inexplicably strong SEP event (or sequence of smaller events), providing the first definite evidence for a SEP event of this magnitude from multiple datasets.

- This interpretation is in agreement with enhanced auroral sightings reported in historical chronicles for the period.

Acknowledgements. G.A.K. was partly supported by the Program No. 22 presidium RAS and by the Academy of Finland.

\section{References}

Allen, J. 2012, Nature, 486, 473

Beer, J. 2000, Space Sci. Rev., 94, 53

Beer, J., McCracken, K., \& von Steiger, R. 2012, Cosmogenic Radionuclides: Theory and Applications in the Terrestrial and Space Environments (Berlin: Springer)

Dall'Olmo, U. 1980, J. History Astron., 11, 10

Damon, P. E., \& Peristykh, A. N. 2004, in Solar Variability and its Effects on Climate. Geophysical Monograph 141, eds. J. M. Pap, et al. (Washinton DC: AGU), AGU Geophys. Monog. Ser., 141, 237

Damon, P., Lerman, J., \& Long, A. 1978, Ann. Rev. Earth Planet. Sci., 6, 457

Dergachev, V., \& Veksler, V. 1991, Application of the Radiocarbon Method for Studies of the Environment in the Past (A.F. Ioffe Phys-Tech Inst., Acad. Sci. USSR, Leningrad, USSR), in Russian

Dorman, L. 2004, Cosmic Rays in the Earth's Atmosphere and Underground (Dordrecht: Kluwer Academic Publishers)

Eichler, D., \& Mordecai, D. 2012, ApJ, 761, L27
Friedrich, M., Remmele, S., Kromer, B., et al. 2004, Radiocarbon, 46, 1111

Goslar, T. 2001, Radiocarbon, 43, 743

Hambaryan, V., \& Neuhäuser, R. 2013, MNRAS, 429

Hogg, A., Palmer, J., Boswijk, G., Reimer, P., \& Brown, D. 2009, Radiocarbon, 51,1177

Horiuchi, K., Ohta, A., Uchida, T., et al. 2007, Nucl. Inst. Meth. Phys. Res. B, 259,584

Horiuchi, K., Uchida, T., Sakamoto, Y., et al. 2008, Quat. Geochronol., 3, 253

Hudson, H. S. 2010, Nature Phys., 6, 637

Keimatsu, M. 1973, A chronology of aurorae and sunspots observed in China, Korea and Japan, Part IV (Ann. Sci. College of Liberal Arts, Kanazawa University), 13

Kovaltsov, G., \& Usoskin, I. 2010, Earth Planet. Sci. Lett., 291, 182

Kovaltsov, G., Mishev, A., \& Usoskin, I. 2012, Earth Planet. Sci. Lett., 337, 114

Link, F. 1962, Geofysikální Sborník, 173, 297

Mac Airt, S., \& Mac Niocaill, G., eds. 1983, The Annals of Ulster (to A.D. 1131) (Dublin: Dublin Institute for Advanced Studies)

McCracken, K. 2004, J. Geophys. Res., 109

Melott, A. L., \& Thomas, B. C. 2012, Nature, 491, E1

Miyake, F., Nagaya, K., Masuda, K., \& Nakamura, T. 2012, Nature, 486, 240

Oeschger, H., Siegenthaler, U., Schotterer, U., \& Gugelmann, A. 1975, Tellus, 27,168

Reimer, P. J., Baillie, M. G. L., Bard, E., et al. 2009, Radiocarbon, 51, 1111

Scholz, B. W., \& Rogers, B. 1970, Carolingian chronicles: Royal Frankish Annals and Nithard's Histories (Ann Arbor: University of Michigan Press)

Schrijver, C. J., Beer, J., Baltensperger, U., et al. 2012, J. Geophys. Res., 117, A08103

Siegenthaler, U., Heimann, M., \& Oeschger, H. 1980, Radiocarbon, 22, 177

Smart, D. F., Shea, M. A., Spence, H. E., \& Kepko, L. 2006, Adv. Space Res., 37,1734

Swanton, M. 2000, The Anglo-Saxon Chronicles (London: Phoenix Press)

Usoskin, I. G. 2008, Liv. Rev. Sol. Phys., 5

Usoskin, I. G., \& Kovaltsov, G. A. 2012, ApJ, 757, 92

Usoskin, I., Solanki, S., Kovaltsov, G., Beer, J., \& Kromer, B. 2006, Geophys. Res. Lett., 33, L08107

Vonmoos, M., Beer, J., \& Muscheler, R. 2006, J. Geophys. Res., 111, A10105

Xu, Z., Pankenier, D., \& Jiang, Y. 2000, East Asian archaeoastronomy: historical records of astronomical observations of China, Japan and Korea (Amsterdam: Gordon and Breach Science Publishers)

Yau, K., Stephenson, F., \& Willis, D. 1995, A catalogue of auroral observations from China, Korea and Japan (193 B.C. - A.D. 1770), Technical Report RAL-TR-95-073 (Chilton, Oxfordshire: Rutherford Appleton Laboratory)

Yiou, F., Raisbeck, G., Baumgartner, S., et al. 1997, J. Geophys. Res., 102, 26783 